\title{
Reliability and Accuracy of Ball Speed During Different Strokes in Young Tennis Players
}

()(1) $\ominus$

\author{
Authors \\ Irene González-González, 2, David Rodríguez-Rosell³, David Clavero-Martín², Ricardo Mora-Custodio ${ }^{3}$, \\ Fernando Pareja-Blanco ${ }^{3}$, Juan Manuel Yáñez García ${ }^{3}$, Juan José González-Badillo3
}

\author{
Affiliations \\ 1 Fundación San Pablo Andalucía CEU, Bormujos, Spain \\ 2 Andalusian Tennis Federation, Seville, Spain \\ 3 Centro de Investigación en Rendimiento Físico y \\ Deportivo, Universidad Pablo de Olavide, Seville, Spain
}

\author{
Key words \\ groundstrokes, serve, tennis, height, body weight, \\ sport level \\ received $\quad 02.04 .2018$ \\ revised 26.06.2018 \\ accepted 27.06.2018

\section{Bibliography} \\ DOI https://doi.org/10.1055/a-0662-5375 \\ Sports Medicine International Open 2018; 2: E133-E141 \\ (c) Georg Thieme Verlag KG Stuttgart · New York \\ ISSN 2367-1890

\section{Correspondence} \\ Mr. David Rodríguez-Rosell, PhD \\ Centro de Investigación en Rendimiento Físico y Deportivo \\ Universidad Pablo de Olavide \\ Ctra. de Utrera, km 1 \\ 41013 Seville \\ Spain \\ Tel.: + 34/622/467 840, Fax: + 34/954/349 238 \\ davidrodriguezrosell@gmail.com
}

\begin{abstract}
This study aimed to examine the relationship between anthropometric and sport level (SL) variables and both ball speed and accuracy in young male competitive tennis players. A secondary aim of this study was to analyze the possible differences in ball speed and accuracy between players of different competitive levels. A total of 85 players (age: $14.7 \pm 2.4$ years; height: $1.65 \pm 0.12 \mathrm{~m}$; body weight: $56.3 \pm 13.4 \mathrm{~kg}$ ) were divided into five groups according to their positions in the ranking list. To measure stroke performances, the goal was to hit 20 balls at the maximum possible speed inside the court, with the opportunity of hitting a maximum of 30 balls for each groundstroke and 40 for serve. Accuracy was calculated by dividing the number of balls inside the default surface by the total number of hits. The ball speed showed high reliability for all three strokes assessed. The ball speed progressively increased as SL increased for all strokes, whereas accuracy remained unchanged across SL groups. All independent variables presented significant relationships $(r=0.59-0.85, p<0.05-0.001)$ with ball speed for all strokes. However, after applying partial correlations these relationships decreased substantially $(r=0.02-$ $0.51)$. The accuracy showed significant relationships with $\mathrm{SL}$ only in the serve $(r=0.31, p<0.05)$ and backhand $(r=0.26$, $\mathrm{p}<0.05)$ strokes. In conclusion, the results of the present study suggest that tennis performance depends on increasing ball speed while maintaining a relatively stable level of accuracy.
\end{abstract}

\section{Introduction}

Tennis is a sport that demands a complex interaction of several physical, physiological and anthropometric components for successful performance $[3,11,31]$. In this regard, ball speed is usually considered one of the most important attributes of performance in tennis [19,31], because an increased stroke speed reduces the time for an opponent to prepare to return the ball successfully. In fact, it appears that the evolution within the game has been characterized by a progressive increase in the ball speed during groundstrokes and serves [8], which may be a consequence of developing the physical, technical and tactical preparation of tennis players.
In addition, ball speed seems to be the main factor differentiating high-level tennis players from those of lower levels, regardless of the age of the players $[19,30,32]$. For these reasons, players generally trying to maximize the ball speed of groundstrokes and serve without sacrificing accuracy $[19,31]$. Therefore, because the importance of these variables for high performance in professional and young tennis players [19, 30,31], it appears necessary to know the ball speed and accuracy during different strokes, how these variables evolve with the increasing sport level (SL), as well as the factors impacting speed and accuracy.

Most studies analyzing tennis strokes have focused on the biomechanical aspects of the player's movement and influence of rack- 
et characteristics $[1,4,14,25,26]$. In addition, several studies $[3,8,31]$ have examined the influence of anthropometric characteristics in ball speed and accuracy, mainly during the serve. Results of these studies $[3,8,31]$ appear to indicate that height plays a determining role in both variables during the serve. Theoretically, taller players can achieve higher ball impact height, allowing them to serve at a higher speed than smaller players with the same probability of a successful serve [31]. In this connection, significant positive relationships between height and ball speed during the serve have been observed in professional tennis players $[3,8,31]$. Conversely, no significant relationship has been found between serve ball speed and body weight [3]. However, the specific role of other anthropometric variables such as wingspan on ball speed during the serve, as well as the possible influence of height, body weight and wingspan on ball speed in groundstrokes still remains unanalyzed. Similarly, the above-mentioned studies $[3,8,19,31]$ were conducted with adult tennis players, whereas little is known about the relationship between these anthropometric variables and the performance (ball speed) in the different tennis strokes in young players.

In addition to ball speed, successful performance in tennis also depends on accuracy. During the serve, hitting the ball at a greater height allows a larger area of the service box into which the ball can land, so the ball is less likely to land outside the service box and be classed as an error $[5,26]$. This means that the height to which the ball is hit also has a positive influence on the accuracy and success of the serve [31]. However, the relationship between accuracy and height or other physical characteristics seem to have not been sufficiently studied. Moreover, contrary to what one may think, ball speed does not seem to have a negative influence on accuracy in tennis. Studies on this matter $[4,6,13,19]$ have shown that there was no significant difference in accuracy as a consequence of higher ball speeds in the different strokes in tennis, indicating that: (1) the more powerful shots of the highly skilled tennis players are as accurate as their slower shots [6]; and (2) the high-level players show equally or even more hitting accuracy than players of a lower level [19]. In fact, a relationship between ball accuracy and the level of tennis performance have been shown in previous studies $[19,20]$. This means that experienced or high-level tennis players try to maintain a high racquet speed throughout their strokes, because otherwise it would probably result in unsuccessful hits due to changed timing patterns or in an easy shot for their opponent $[7,19]$.

Despite the considerable high demands of elite tennis and the increased participation in competitions from an early age, studies conducted with young tennis players are scarce and have been mainly focused on analyzing the evolution of different anthropometric and fitness characteristics [16-18, 22, 23, 30]. However, combined data on speed and accuracy in forehand, backhand and serve strokes of young tennis players with different SL are limited $[15,30]$. Therefore, although a number of studies have been focused on young tennis players, more information is needed about the contribution of various anthropometric characteristics in oncourt tennis play in this population, especially in relation to the ball speed and the accuracy of the different strokes. In light of the above considerations, the purposes of this study were: (1) to compare ball speed and accuracy of groundstrokes (forehand and backhand) and serve between young tennis players of different SL; and (2) to examine which anthropometric characteristics and other variables such as age and competitive level could best explain both ball speed and ball placement as criterion measures in young male competitive tennis players.

\section{Methods}

\section{Participants}

A total of 85 male tennis players [age, $14.7 \pm 2.4$ years; height, $1.65 \pm 0.12 \mathrm{~m}$; body weight (BW), $56.2 \pm 13.32 \mathrm{~kg}$; wingspan, $1.68 \pm 0.14 \mathrm{~m}]$ participated voluntarily in this study. The inclusion criteria were: 1 ) take part of the talent development program of the Regional Tennis Federation, and 2) be on the national ranking lists. The players were divided into five groups according to their $\mathrm{SL}$, which was determined through their positions in the national ranking list, as follows: SL1, national score $\leq 45$ points; SL2, $45<$ national score $\leq 200$ points; SL3, $200<$ national score $\leq 500$ points; $S L 4,500<$ national score $\leq 650$ points; SL5, national score $>650$ points. The total points correspond to the sum of the points obtained in the different national (Spanish) tournaments. Players only played in singles modality. Currently, the points for each player are added and defended each month. Player characteristics are displayed in $>$ Table 1. All tennis players were considered healthy, with

- Table 1 Physical and competitive characteristics of the participants for each sport level group (mean \pm SD).

\begin{tabular}{|l|c|c|c|c|c|}
\hline & $\mathbf{S L 1}(\mathbf{n}=\mathbf{1 4})$ & $\mathbf{S L 2}(\mathbf{n}=\mathbf{2 8})$ & $\mathbf{S L 3}(\mathbf{n}=\mathbf{2 3})$ & $\mathbf{S L 4}(\mathbf{n}=\mathbf{1 0})$ & $\mathbf{S L 5}(\mathbf{n}=\mathbf{1 0})$ \\
\hline Age (years) & $14.3 \pm 1.2^{* * *}$ & $13.7 \pm 2.6^{* * *}$ & $14.5 \pm 1.6^{* * *}$ & $14.9 \pm 1.6^{* *}$ & $18.3 \pm 2.0$ \\
\hline Height (cm) & $164.5 \pm 9.4$ & $157.9 \pm 14.0^{* *}$ & $166.8 \pm 9.8$ & $169.6 \pm 9.6$ & $177.5 \pm 6.0$ \\
\hline BW (kg) & $53.7 \pm 9.7^{* * *}$ & $48.4 \pm 12.4^{* * *} \dagger$ & $57.1 \pm 11.2^{* *}$ & $63.0 \pm 12.3$ & $73.4 \pm 6.1$ \\
\hline Wingspan (cm) & $168.5 \pm 10.1$ & $160.0 \pm 15.5^{* *}$ & $168.8 \pm 10.6$ & $173.1 \pm 11.6$ & $188.6 \pm 8.3$ \\
\hline Maturity offset & $0.55 \pm 1.09^{* * *} \dagger$ & $-0.05 \pm 2.12^{* * *} \dagger$ & $0.79 \pm 1.38^{* * *}$ & $1.18 \pm 1.49^{* *}$ & $3.72 \pm 1.06$ \\
\hline PAS (\%) & $93.0 \pm 4.0^{* * *}$ & $89.9 \pm 7.3^{* * *}$ & $93.4 \pm 4.6^{* * *}$ & $94.6 \pm 4.4^{* * *}$ & $99.6 \pm 0.4$ \\
\hline National score & $30.3 \pm 10.4$ & $116.6 \pm 44.9$ & $352.7 \pm 77.8$ & $573.4 \pm 41.2$ & $695.6 \pm 24.9$ \\
\hline National ranking & $6423.9 \pm 433.8$ & $1838.2 \pm 797.1$ & $901.1 \pm 185.0$ & $376.7 \pm 189.5$ & $102.1 \pm 32.5$ \\
\hline Competitions & $5.5 \pm 2.0$ & $10.5 \pm 1.2$ & $16.4 \pm 1.1$ & $22.7 \pm 1.7$ & $28.7 \pm 1.4$ \\
\hline
\end{tabular}

SL: sport level; BW: body weight; PAS: predicted adult stature; BS: ball speed; S: serve; F: forehand; B: backhand; Competitions: number of competitions per year. Statistically significant differences with respect to SL_5: ${ }^{*} p<0.05,{ }^{* *} p<0.01,{ }^{* * *} p<0.001$. Statistically significant differences with respect to SL_4: ${ }^{\dagger} p<0.05$. Note: The national Score, national ranking position and competitions variables progressively increased $(p<0.001)$ from SL1 to SL5. 
no significant bodily injury at the time of testing, and had been playing tennis consistently for a minimum of 4 years. The players, technical staff, and parents were informed of all experimental procedures and potential risks of the study. Parental/guardian informed consent for all players involved in this investigation were obtained before participation. The present investigation met the ethical standards of this journal [12], was approved by the ethical committee of the Pablo de Olavide University and was consistent with the ethical requirements for human experimentation in accordance with the Declaration of Helsinki.

\section{Study design}

A cross-sectional research design was used to analyze: (1) if the independent variables height, BW, wingspan, age, and SL are related to both the ball speed and accuracy in young male national competitive tennis players; and (2) the differences in these variables between different competitive levels. To address this issue, 85 young male tennis players, who were divided into five groups according to their positions in the national ranking lists, were tested in a single testing session. The battery of tests performed included anthropometrics assessments and ball speed tests during three different strokes: forehand, backhand and serve. For these on-court tests, the goal was to land 20 balls inside the designated area hitting the ball as fast as possible. To do this, the players had the opportunity to hit a maximum of 30 balls in parallel for the groundstrokes (backhand and forehand), and 40 for the serve ( 20 balls to each side of the court). The testing session was conducted right after the end of the season, when participants were not involved in competitive activities. In the preceding 2 weeks of this study, 2 preliminary familiarization sessions were undertaken so that the players could become familiar with the dynamics of the on-court tests.

\section{Testing procedures}

The players were tested using anthropometric and on-court performance variables. The physical characteristics included age, height, BW, and wingspan. The on-court performance tests included the measurement of post-impact ball speed in parallel groundstrokes (backhand and forehand) and post-impact ball speed in crossover serves. The testing session was conducted in the morning (10:00-12:00 am) under similar environmental conditions $\left(\sim 22^{\circ} \mathrm{C}-24^{\circ} \mathrm{C}\right.$ and $\sim 55 \%-65 \%$ humidity) for all participants.

\section{Anthropometric measures}

Height and BW were measured using a medical stadiometer and scale (Seca 710, Ltd., Hamburg, Germany). Wingspan was measured from the tip of one middle finger to the tip of the other middle finger, with the participants abducting both arms horizontally and perpendicular to the body's upright position [24]. The maturity status of the participants was determined using years from/to peak height velocity (PHV) [i. e., maturity offset $=-7.999994+(0.0036124 \times$ age $\times$ height $)$; $\mathrm{R}^{2}=0.896 ; \mathrm{SEE}=0.542$ ] [21], as well as the percentage of predicted adult stature [27]. At least 2 days before test time, there were no fatiguing training sessions. Strong verbal encouragement was provided during all on-court tests to motivate participants to give a maximal effort.

\section{On-court performance}

Forehand, backhand and serve tests were performed on a hardsurface tennis court. All the participants performed the same warm-up protocol, which consisted of 5 min of jogging at an easy pace, 10 min of upper and lower-limb joint mobilization exercises, and 20 hits for forehand, backhand and serve, respectively, at a progressive intensity. Then, the players were allowed to perform 5 maximal practice trials prior to testing. After a 2-min rest, the test began. During the test, participants were instructed to "hit the ball to the target square and hit it as fast as possible" [19]. For the groundstrokes, the same coach, former tennis player, with extensive experience in teaching tennis, threw balls from the middle of the court on the opposite side of the player, leaving the corridor free to allow groundstrokes to be made in parallel ( $>$ Fig. 1). The speed of the ball thrown by the coach was $\sim 50-60 \mathrm{~km} \cdot \mathrm{h}^{-1}$ (recorded using the radar gun). For the groundstrokes, the players were placed standing in the middle of the baseline. They made the proper movement to hit the ball, and after each stroke the participant had to return to the center of the baseline before returning the next ball. The tests were carried out in groups of 3 players. Each participant performed sets of 5 strokes alternating with the other players in the group until they completed the maximum number of strokes allowed ( 30 for groundstrokes or 40 for the serve), or until they achieved the goal of landing 20 balls on the designated area (see - Fig. 1). Thus, after each set, participants had a 1-2 min rest to avoid any effect of fatigue on ball speed or accuracy. The different strokes assessed (forehand, backhand and serve) were carried out in random order for each group of participants. In each stroke, the ball speed was written down when the ball landed inside the court (groundstrokes) or the service box (serve), and was recorded only as a hit when the ball landed outside the designated target area. Two trained researchers were responsible for supervising the landing of each ball and determining whether the ball was "in" or "out." The maximum (the best) ball speed, the mean speed and the number of strokes were recorded for the subsequent analysis. Mean speed was calculated as the average speed of all those balls that landed inside the court.

\section{Accuracy quantification}

Accuracy was calculated by dividing the number of balls inside the defined surfaced by the total number of hits in each type of stroke in relative terms [Accuracy $=$ (Ball “in"/Total hits) $x$ 100]. Therefore, the value obtained from this equation always range from 0 to $100 \%$, in such way that the higher the ratio (i. e., closest to $100 \%$ ), the greater the accuracy.

\section{Measurement equipment}

Post-impact ball speed was measured using a radar gun (Stalker ATS II Professional Sports Radar, Plano, TX, USA; accuracy $\pm 1.6 \mathrm{~km} \cdot \mathrm{h}^{-1}$; range of velocity: from 1.6 to $1287 \mathrm{~km} \cdot \mathrm{h}^{-1}$; frequency: $34.7 \mathrm{GHz}$ ). This device has been validated and used in previous human experiments to measure sprint running $[9,10]$, kicking [29] and throwing the ball [28]. For the groundstrokes, the researcher in charge of measuring the ball speed held the radar gun and stood about $2 \mathrm{~m}$ behind the player adjusting position according to the point where the player hit the ball, and with the radar 


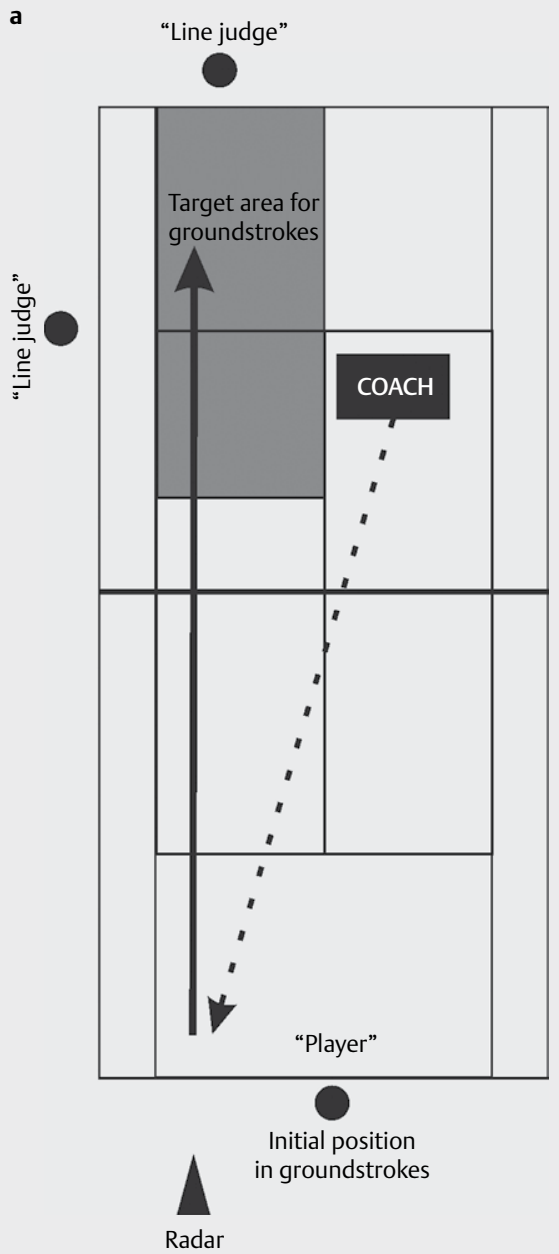

b

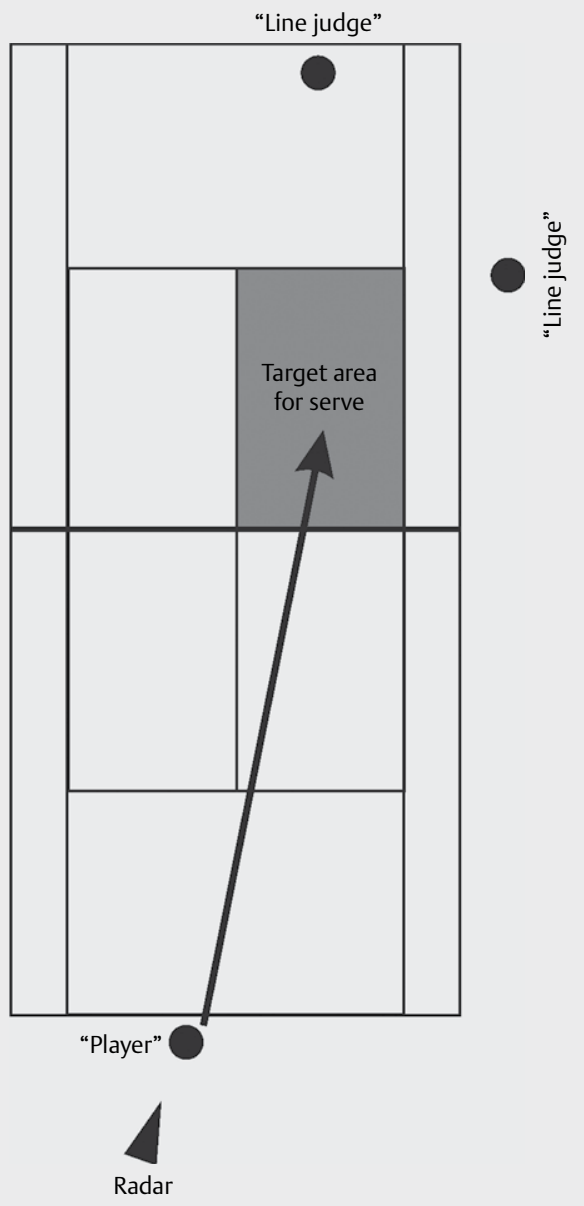

- Fig. 1 Experimental set-up for groundstrokes $\mathbf{a}$ and serve $\mathbf{b}$.

axis aligned in the direction and height of the ball trajectory (according to radar gun specifications). Another experienced researcher observed and wrote the speed down if the ball landed inside the court. For the serve, the researcher was placed on a platform $2 \mathrm{~m}$ in height holding the radar gun with the radar axis aligned in the direction of the ball trajectory.

\section{Statistical analysis}

The data obtained are presented as mean \pm SD. A one-way random effects model with ANOVA analysis was applied to calculate the intraclass-correlation coefficient (ICC, model 2,1) to determine relative reliability [34]. The size of the correlation evaluated as follows: $r<0.7$ low; $0.7 \leq r<0.9$ moderate and $r \geq 0.9$ high [33]. Absolute reliability was reported using the standard error of measurement (SEM) [34], which was calculated as the square root of the intra-

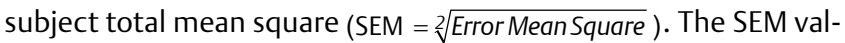
ues were expressed in relative terms through the coefficient of variation (CV) [2]. The maximum amount of the standard error of measurement differences, which we express as the minimal difference (MD) between two measurements to consider these measurements as different, was calculated by multiplying the standard error of measurement differences (SEM $\cdot \sqrt{2}$ ) by 1.96 (confidence interval $=95 \%$ ), resulting the following equation: $M D=\operatorname{SEM} \cdot \sqrt{2 \cdot 1.96}$. Because the relative effects of maturational status would exists on both anthropometric and technical characteristics, an analysis of covariance (ANCOVA) controlling for the maturity offset variable was then used with Bonferroni's correction to detect differences between the groups in anthropometric variables, ball speed and accuracy. This analysis creates an adjusted mean for values of all five groups depending on the maturity status. A one-way analysis of variance (ANOVA) with Bonferroni's post-hoc pairwise comparisons was used to detect differences between the different strokes (serve, forehand and backhand) in ball speed and accuracy variables. Pearson's correlation coefficients were calculated to establish the relationships between the variables analyzed. To detect possible spurious relationships, partial correlations between each variable and the ball speed were calculated by controlling the rest of the variables. A stepwise multiple regression analysis was conducted to determine the importance of the variables assessed in predicting ball speed in the three strokes, and the regression model assumptions were analyzed. All data analyses were performed using SPSS (V17.0; SPSS, Inc., Chicago, IL, USA). 


\section{Results}

\section{Ball speed}

The ANCOVA test revealed that the maturity status showed a significant effect $(p<0.05)$ on ball speed, but the SL factor also resulted in a significant effect in all the strokes (serve: $F=6.73, p<0.001$; forehand: $F=5.93, p<0.001$; backhand: $F=8.37, p<0.001$ ). The ball speed increased progressively from the SL_1 to SL_ 5 group in all the three strokes assessed, with SL_4 and SL_5 showing significantly greater ball speed than SL_1 and SL_2 ( $>$ Fig. 2). In addition, SL_5 had a tendency to show higher ball speed values than $S L \_3$ in the serve $(p=0.07)$, forehand $(p=0.08)$ and backhand $(p=0.08)$ strokes. All the groups showed significantly $(p<0.05)$ higher ball speed during serve compared to forehand and backhand strokes, whereas ball speed during the forehand was always significantly $(p<0.05)$ greater than the backhand stroke ( $\mathbf{F i g} .2)$.

\section{Accuracy}

The ANCOVA test revealed that the maturity status had no effect on accuracy (serve: $F=0.026, p=0.87$; forehand: $F=3.143, p=0.08$; backhand: $F=0.994, p=0.32$ ). There were no significant differences between SL groups in accuracy for any stroke assessed, except for SL_1 with SL_3 and SL_ 5 in the serve stroke. All groups resulted in significantly greater $(p<0.001)$ accuracy in groundstrokes than the serve stroke ( $\vee$ Fig. 3 ).

\section{Reliability of ball speed}

The maximum ball speed showed high absolute (CV) and relative (ICC) reliability scores for the three types of strokes in both when the data were analyzed by groups ( $\triangleright$ Fig. 4 ) and when the data was pooled ( $\triangleright$ Table 2 ). The serve was the most reliable stroke in all SL groups, with ICC values higher than 0.94 and CV values ranging from 2.3-6.2\% ( $>$ Fig. 4). Reliability scores for forehand and backhand strokes were very similar ( $>$ Table 2 , $>$ Fig. 4 ). The reliability for each SL group in the same variables was similar, with systematically lower CV in SL_5 in all cases. In addition, both the ICC and CV of the first and last five strokes were similar for all three strokes assessed and all the SL groups (• Fig. 4).

\section{Relationship between anthropometric variables and ball speed and accuracy}

Significant correlations ( $p<0.001$, in all cases) were observed between the maximum and mean ball speed in the three strokes and the variables age, SL, BW, height, wingspan and maturity offset ( $\triangleright$ Table 3). When these correlations were controlled for the rest of the variables, the correlation coefficient values were considerably reduced, with only the SL variable maintaining significant correlations $(p<0.001)$ with ball speed in all the three strokes assessed.

A multiple regression analysis was conducted to determine the importance of age, SL, BW, height, maturity offset and wingspan in predicting ball speed performance on the three strokes ( $\triangleright$ Table 4). The explained variance $\left(R^{2}\right)$ was slightly higher for the maximum ball speed than for the mean speed in all three strokes, with a tendency to be higher in the serve compared to the forehand and backhand strokes. The SL was the only variable present in all the regression equations. The regression model assumptions were satisfactorily ful-

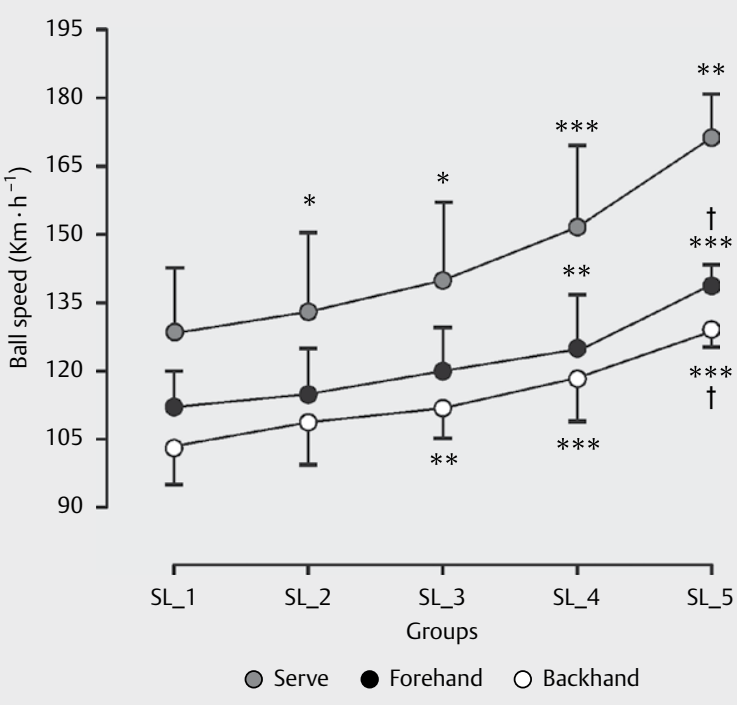

- Fig. 2 Mean speed of the ball in each stroke according to sport level (SL). Statistically significant differences compared to SL_1: ${ }^{*} \mathrm{p}<$ $0.05,{ }^{* *} \mathrm{p}<0.01,{ }^{* * *} \mathrm{p}<0.001$. Statistically significant differences compared to SL_2: ${ }^{\dagger} p<0.05$. Note: Mean ball speed during the serve was significantly $(p<0.001)$ greater than both groundstrokes for all the sport level groups, whereas mean ball speed during the forehand was significantly $(p<0.001)$ greater than the backhand stroke for all the sport level groups.

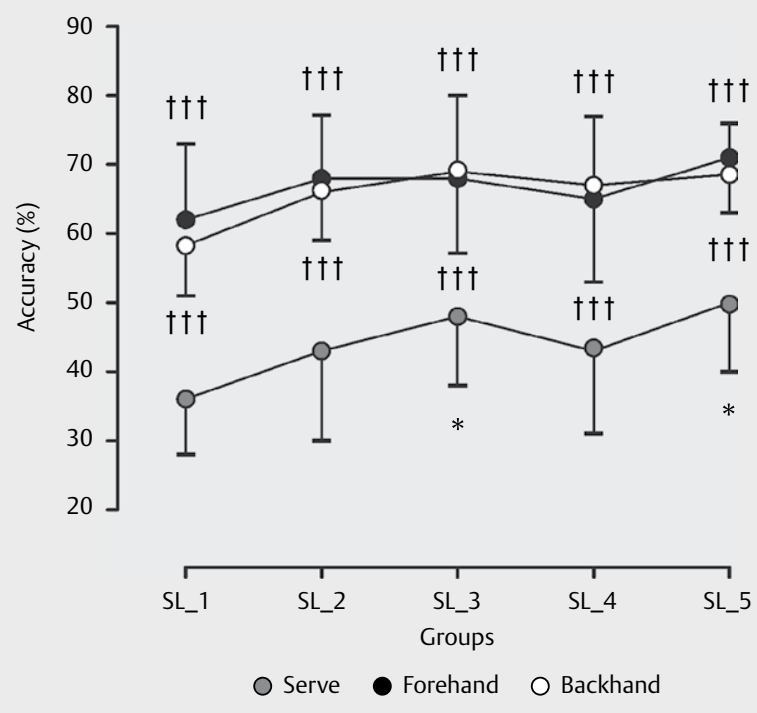

- Fig. 3 Accuracy in each stroke according to sport level (SL). Statistically significant differences compared to $S L_{-} 1:{ }^{*} p<0.05$. Statistically significant differences compared to the serve: $\mathrm{tt \dagger p}<0.001$.

filled. All values of the Durbin-Watson (DW) independence test were close to 2. Similarly, all values of tolerance were higher than 0.1 and the variance inflation factor (VIF) lower than 10 . In all cases, the dependent variable was adjusted to normality.

The accuracy showed only weak significant relationships with SL in the serve $(r=0.31, p<0.05)$ and backhand $(r=0.26, p<0.05)$ 

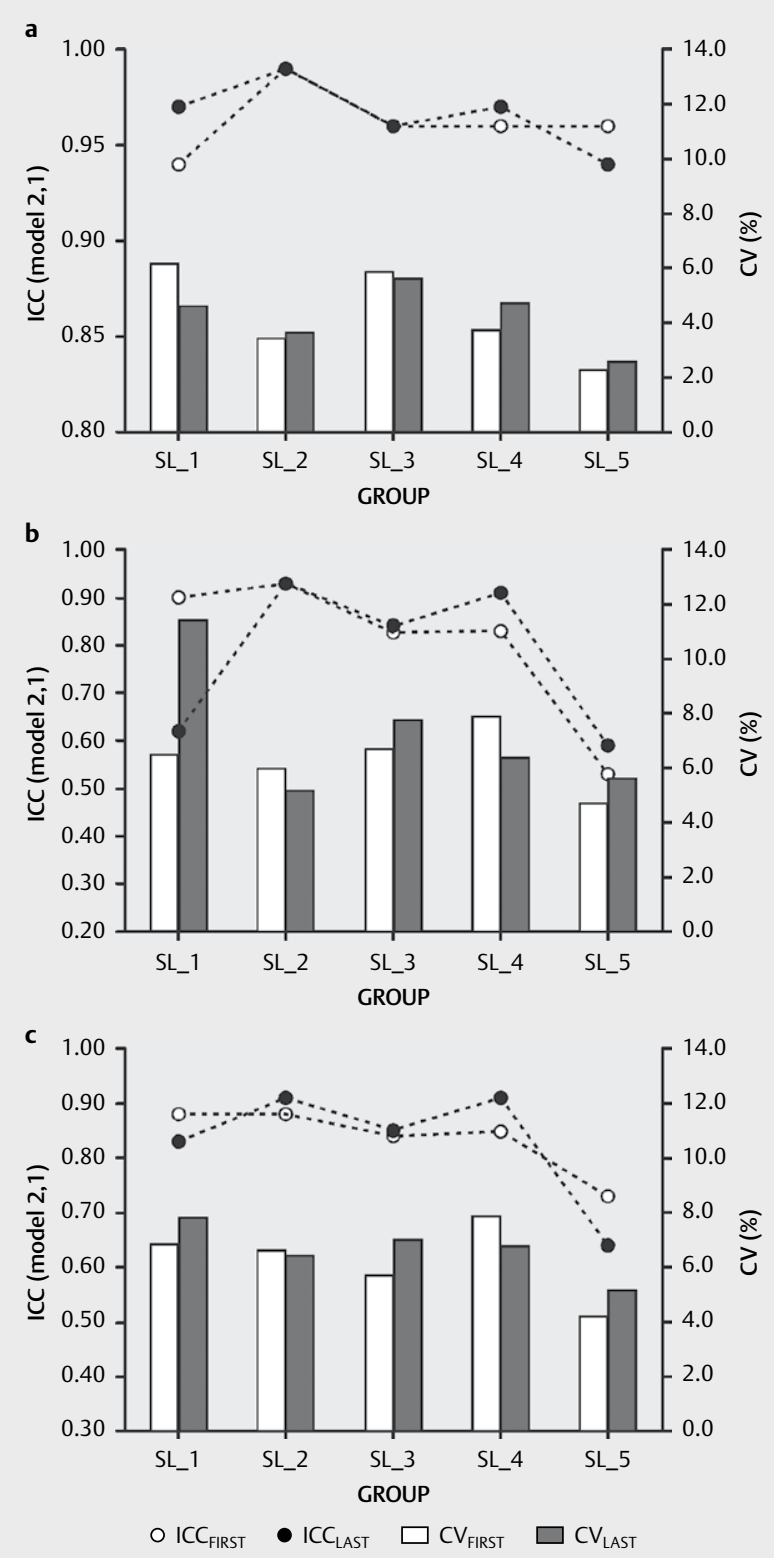

-Fig. 4 Reproducibility of ball speed measurements for the serve $\mathbf{a}$, forehand $\mathbf{b}$ and backhand $\mathbf{c}$ stroke. The ICC $(2,1)$ and CV values for the first and last five strokes in each group are reported.

strokes. No significant relationships were found with any other anthropometric variable. In addition, the accuracy also showed no relationship either with the mean or maximum ball speed in any stroke assessed.

\section{Discussion}

In the present study, we compared the ball speed and accuracy in young tennis players with different SL, and we also analyzed the potential impact of age, SL, BW, height, maturity status and wingspan on ball speed during groundstrokes and serve. The main find- ings of this study were: 1 ) ball speed showed a high reliability for all three strokes assessed, regardless of the SL, with a tendency to show greater reliability scores in the serve compared to the forehand and backhand; 2) the ball speed increased progressively from SL_1 to SL_5 in all three strokes, however the accuracy remained practically stable between the different SL groups; 3) all variables (age, SL, BW, height, maturity status and wingspan) showed significant correlations with ball speed in all three strokes, but after applying partial correlation in order to control the influence of the rest variables in the relationships, these correlation coefficients decreased substantially; and 4) some of the above-mentioned variables (mainly BW and SL) explained a remarkable percentage of variance (from 57.7 to $81.4 \%$ ) of mean and maximum ball speed when multiple linear regressions were applied.

\section{Reliability}

All five SL groups showed a high absolute (CV: 4.37-7.30\%) and relative (ICC: $0.90-0.98$ ) reliability scores in ball speed for all types of strokes in both the first and last five strokes ( $>$ Table 2 ). Previous studies have shown similar (ICC: 0.87-0.99) [17] or even lower (ICC: 0.748 , $95 \%$ confidence interval: $0.149-0.945$ ) [3] reliability scores in tennis players of different ages. Comparison between different strokes indicated that reliability tended to be better in the serve compared to the forehand and backhand. These results are especially relevant taking into account that the serve is considered a stroke of greater technical difficulty than groundstrokes. Thus, this tendency could be due to the greater stability in the position of the subject and ball speed before hitting in the serve compared to the groundstrokes, because the player himself places the ball in the proper height and position, whereas in the forehand and backhand strokes the player has to adapt the stroke to the height and speed of the approaching ball. In addition, our results showed similar reliability scores for all SL groups ( $\mathbf{F i g}$. 4). Therefore, this generally high stability of ball speed found in all the three strokes assessed suggests that we can have considerable confidence in the rest of the results of this study.

\section{Accuracy}

In the present study, a greater accuracy level meant that the player needed fewer strokes to place 20 balls inside the target area ( $\vee$ Fig. 1). Our results showed that although the ball speed showed a clear tendency to increase with the SL ( $\vee$ Fig. 2 ), the accuracy remained practically stable for all SL groups ( $>$ Fig. 3). Only SL_5 and SL_3 showed a greater accuracy level $(p<0.05)$ than $S_{-} 1$ in the serve, whereas no significant differences were found in either of the two groundstrokes assessed ( $>$ Fig. 3). This tendency has been previously observed during the serve $[4,6]$, with no decrease in accuracy when the ball speed increased. In addition, similar to our results, a previous study [19] found that the ball speed during the forehand and backhand was higher for elite players compared to high-performance tennis players, however both groups showed similar ability in terms of the accuracy. Therefore, these results suggest that the increase in tennis performance is highly dependent on increasing ball speed with relatively stable accuracy, especially for the forehand and backhand strokes. 
- Table 2 Reliability of ball speed measurements in the serve, forehand and backhand strokes for all groups pooled.

\begin{tabular}{|c|c|c|c|c|c|c|}
\hline & ICC (Cl: 95\%) & SEM $\left(\mathbf{k m} \cdot \mathbf{h}^{-1}\right)$ & CV (\%) & $\mathrm{MD}\left(\mathrm{km} \cdot \mathrm{h}^{-1}\right)$ & Mean $\left(\mathrm{km} \cdot \mathrm{h}^{-1}\right)$ & MD (\%) \\
\hline \multicolumn{7}{|l|}{ Serve } \\
\hline F_5_S & $0.98(0.97-0.99)$ & 6.40 & 4.50 & 17.73 & 140.96 & 12.58 \\
\hline L_5_S & $0.98(0.97-0.99)$ & 6.17 & 4.37 & 17.10 & 141.27 & 12.10 \\
\hline \multicolumn{7}{|c|}{ Forehand } \\
\hline F_5_S & $0.92(0.89-0.95)$ & 7.50 & 6.36 & 10.60 & 118.44 & 17.55 \\
\hline L_5_S & $0.90(0.87-0.93)$ & 8.90 & 7.30 & 24.67 & 122.07 & 20.21 \\
\hline \multicolumn{7}{|c|}{ Backhand } \\
\hline F_5_S & $0.91(0.88-0.94)$ & 7.10 & 6.30 & 19.68 & 112.18 & 17.58 \\
\hline L_5_S & $0.92(0.89-0.94)$ & 7.50 & 6.67 & 20.79 & 112.60 & 18.46 \\
\hline
\end{tabular}

- Table 3 Pearson's correlation coefficients between mean ball speed and different variables, and partial correlations controlling for the rest of variables in the three strokes: serve, forehand, and backhand.

\begin{tabular}{|c|c|c|c|c|c|c|c|c|c|}
\hline & \multicolumn{3}{|c|}{ Serve } & \multicolumn{3}{|c|}{ Forehand } & \multicolumn{3}{|c|}{ Backhand } \\
\hline & $\mathbf{r}$ & $r_{\text {partial }}$ & $\operatorname{LEV}(\%)$ & $\mathbf{r}$ & $r_{\text {partial }}$ & $\operatorname{LEV}(\%)$ & $\mathbf{r}$ & $r_{\text {partial }}$ & $\operatorname{LEV}(\%)$ \\
\hline Age & $0.79^{* * *}$ & $0.35^{* * *}$ & 81.0 & $0.69^{* * *}$ & $0.23^{*}$ & 89.5 & $0.62^{* * *}$ & 0.16 & 94.7 \\
\hline SL & $0.59^{* * *}$ & $0.39^{* * *}$ & 57.1 & $0.63^{* * *}$ & $0.44^{* * *}$ & 52.5 & $0.66^{* * *}$ & $0.51^{* * *}$ & 39.5 \\
\hline BW & $0.85^{* * *}$ & $0.39^{* * *}$ & 79.1 & $0.71^{* * *}$ & 0.10 & 98.0 & $0.66^{* * *}$ & 0.08 & 100 \\
\hline Height & $0.78^{* * *}$ & 0.13 & 91.7 & $0.68^{* * *}$ & 0.11 & 97.1 & $0.60^{* * *}$ & 0.03 & 99.7 \\
\hline Wingspan & $0.76^{* * *}$ & 0.10 & 100 & $0.66^{* * *}$ & 0.02 & 100 & $0.61^{* * *}$ & 0.10 & 100 \\
\hline Maturity offset & $0.83^{* * *}$ & 0.19 & 94.2 & $0.73^{* * *}$ & 0.15 & 96.2 & $0.66^{* * *}$ & 0.09 & 98.1 \\
\hline PAS (\%) & $0.24^{*}$ & 0.06 & 93.3 & $0.25^{*}$ & 0.16 & 66.7 & 0.21 & 0.09 & 80.0 \\
\hline
\end{tabular}

\section{Correlations between age, SL, BW, height, wingspan and ball speed}

Significant correlations were observed between the mean and maximum ball speed in the three strokes and age, SL, BW, height, and wingspan ( $>$ Table 3 ). In agreement with our results, previous studies $[3,8,19,31]$ also have shown the positive influence of height and wingspan on ball speed during the serve. As a novel finding, our results suggest that these variables are also relevant in the forehand and backhand strokes. Thus, the correlations values appear to indicate that all these anthropometric and sport level variables are very important to yield high performance in young tennis players. However, partial correlation analysis showed that these individual relationships are highly influenced by the other variables. For example, in the serve stroke, after applying partial correlations only age, SL, and BW maintained a significant correlation with ball speed $(p<0.001)$, but with a loss of explained variance ranging from 57 to $81 \%$ ( $\triangleright$ Table 3$)$. In the forehand, age $(p<0.05)$ and SL $(p<0.001)$ maintained a significant relationship with ball speed, whereas in the backhand only SL showed a significant correlation $(p<0.001)$ after applying partial correlations ( $\triangleright$ Table 3 ). The partial correlations between ball speed and the rest of the variables for all three strokes showed values close to zero ( $\triangleright$ Table 3 ). Therefore, it appears that after applying partial correlations analysis, only the SL variable maintained a significant correlation with ball speed in all three strokes. These results are in accordance with the previous suggestion indicating that progression to excellence in tennis is highly dependent on increase ball speed, because only SL showed a significant independent relationship with this parameter, and with a lower loss of explained variance compared to the rest of the variables. These results represent an important and novel finding and suggest that caution should be taken when determining what variables are important for tennis performance. For example, several studies have shown a significant relationship between height and ball speed during the serve $[3,8,30,31]$, and groundstrokes $[14,19]$, but in these studies the influence of other variables (BW, SL, wingspan) was not controlled. Therefore, our results seem to indicate that it is necessary to analyze the partial correlations in order to know the real influence of each variable on the ball speed during different strokes in tennis.

\section{Explained variance of ball speed through multiple regression analysis}

The multiple regression analysis showed slightly higher explained variance $\left(R^{2}\right)$ for the maximum ball speed than for the mean speed in the three strokes, with a tendency to be higher in the serve compared to the forehand and backhand ( $>$ Table 4 ). The greater explained variance in the serve may be in relation to the higher stability in this type of stroke. For the serve, the three variables in the equations were the same (BW, SL, age) for mean and maximum ball speeds, and they correspond with the three variables that exhibited greater independence when the partial correlation was applied ( Table 3). Thus, the results of multiple regression analysis reinforce the results obtained in the partial correlation study. Surprisingly, al- 
- Table 4 Statistics of multiple linear regression with stepwise method and mean and maximum ball speed as dependent variables in the three strokes.

\begin{tabular}{|c|c|c|c|c|c|c|c|}
\hline Dependent variable & $\mathbf{R}^{2}$ & DW & Equation & SC & Toler & VIF & K-S \\
\hline \multirow[t]{3}{*}{ Mean BS in the serve } & \multirow[t]{3}{*}{0.793} & \multirow[t]{3}{*}{1.69} & \multirow[t]{3}{*}{$0.77 \mathrm{BW}+3.77 \mathrm{SL}+0.73 \mathrm{age}+47.7$} & BW: 0.50 & 0.358 & 2.79 & \multirow[t]{3}{*}{$\mathrm{p}=0.853$} \\
\hline & & & & SL: 0.22 & 0.771 & 1.30 & \\
\hline & & & & Age: 0.30 & 0.380 & 2.57 & \\
\hline \multirow[t]{3}{*}{ Mean BS in the forehand } & \multirow[t]{3}{*}{0.625} & \multirow[t]{3}{*}{2.07} & \multirow[t]{3}{*}{$0.29 B W+3.29 S L+1.45 a g e+73.37$} & BW: 0.32 & 0.354 & 2.825 & \multirow[t]{3}{*}{$p=0.969$} \\
\hline & & & & SL: 0.33 & 0.748 & 1.337 & \\
\hline & & & & Age: 0.28 & 0.380 & 2.633 & \\
\hline \multirow[t]{2}{*}{ Mean BS in the backhand } & \multirow[t]{2}{*}{0.577} & \multirow[t]{2}{*}{1.91} & \multirow[t]{2}{*}{$0.36 \mathrm{BW}+3.96 \mathrm{SL}+81.53$} & BW: 0.44 & 0.754 & 1.326 & \multirow[t]{2}{*}{$p=0.653$} \\
\hline & & & & SL: 0.44 & 0.754 & 1.326 & \\
\hline \multirow[t]{3}{*}{ Maximum BS in the serve } & \multirow[t]{3}{*}{0.814} & \multirow[t]{3}{*}{1.9} & \multirow[t]{3}{*}{$0.76 \mathrm{BW}+4.4 \mathrm{SL}+2.37 \mathrm{age}+60.2$} & BW: 0.51 & 0.358 & 2.79 & \multirow[t]{3}{*}{$p=0.895$} \\
\hline & & & & SL: 0.26 & 0.771 & 1.30 & \\
\hline & & & & Age: 0.28 & 0.389 & 2.57 & \\
\hline \multirow[t]{3}{*}{ Maximum BS in the forehand } & \multirow[t]{3}{*}{0.728} & \multirow[t]{3}{*}{1.9} & \multirow[t]{3}{*}{$4.23 \mathrm{SL}+1.69 \mathrm{age}+0.34 \mathrm{WS}+36.6$} & SL: 0.39 & 0.804 & 1.243 & \multirow[t]{3}{*}{$p=0.86$} \\
\hline & & & & Age: 0.30 & 0.403 & 2.481 & \\
\hline & & & & WS: 0.35 & 0.429 & 2.332 & \\
\hline \multirow[t]{2}{*}{ Maximum BS in the backhand } & \multirow[t]{2}{*}{0.656} & \multirow[t]{2}{*}{2.2} & \multirow[t]{2}{*}{$4.96 S L+0.37 W S+47.65$} & SL: 0.521 & 0.86 & 1.162 & \multirow[t]{2}{*}{$p=0.66$} \\
\hline & & & & WS: 0.455 & 0.86 & 1.162 & \\
\hline
\end{tabular}

though previous studies $[3,8,31]$ have indicated that the height of the tennis players is a key factor to obtain a high ball speed during the serve, this variable did not appear in the equation for this stroke neither for the forehand or backhand. However, this does not mean that height is irrelevant for the different strokes, especially for the serve. Our results suggest that this variable has less effect on ball speed in relation to the other variables when all of them are considered together as predictor variables. The absence of the height variable in the equations can be explained by its strong relationship with $B W(r=0.88$; $p<0.001$ ), which could indicate that BW explains almost all the variance that could be attributed to the height variable. In order to check the possible contribution of height to explain the variance of the maximum ball speed, we removed BW as a predictor variable and found that in this case height came into the equation, together with SL and age, but with less explained variance $(78.5 \%)$ and a lower standardized coefficient ( 0.399 ) for height than BW in the equation $(81.7 \%$ and 0.51 , respectively). These alternative analyses suggest that BW has greater predictive power than height. A similar explanation could apply to the fact that wingspan appeared only as a predictor variable for maximum ball speed in the forehand and backhand strokes, but not in the serve, in accordance with previous studies [24]. In contrast, SL is the only variable that appears in all the regression equations, which confirms the suggestion that SL is the variable with a greater independent relationship with the ball speed in all three strokes. Finally, our results also seem to suggest that the backhand is the most complex stroke, because this type of stroke showed a lower explained variance and equations included a smaller number of variables compared to the serve and forehand strokes ( Table 4).

\section{Limitations}

The current study presents mainly two limitations. First, players were not limited to a particular stance, grip or technique. Moreover, each player used his own racquet and differences in dimensions and string tension of the players' racquets were not assessed. This aspect was a limitation of the present study, because the racquet characteristics (weight, length, area of the striking surface, string tension) can influence the rebound speed of the tennis ball (Bower \& Cross, 2008). However, players reported a preferred string tension between 24 and $28 \mathrm{~kg}$ before testing. On the other hand, during groundstrokes, the balls were thrown by an experienced coach and not by a ball machine. This factor could have influence on ball speed and direction and, consequently, on the ball speed, stability and accuracy during the hit by the player. However, as indicated in methods section, the balls were always thrown by the same trained coach and throwing speed of the ball was stable, ranging from $50-60 \mathrm{~km} \cdot \mathrm{h}^{-1}$. In this way an attempt was made to limit the "coach" effect on ball throwing. In fact, both groundstrokes (forehand and backhand) showed a high absolute and relative reliability. In addition, it is important to indicate that during a tennis match, the ball does not always reach the same place or at the same speed, so the players have to adapt to be able to hit properly in each stroke. Therefore, our study could constitute a more accurate approximation of the analysis of reliability and accuracy in real game conditions.

\section{Practical applications}

Several critical implications for coaches may be derived from this investigation. Firstly, because the ball speed increased progressively as the SL increased in all strokes, the tests for the detection of talents in tennis players should include the measurement of ball speed during different strokes. In addition, of this result it can also be deduced that, in young tennis players, the most important goal in the process of teaching tennis should be increasing ball speed as a priority compared to accuracy. Finally, the results of partial correlations in the current study suggest that further studies are needed in order to determine the real influence of different anthropometric and physical variables on ball speed during the serve and groundstrokes. 


\section{Conclusions}

The main findings of the present study were: 1 ) in tennis players aged 13 to 18 years, the way to excellence is highly dependent on increasing ball speed while maintaining relatively stable accuracy, especially in forehand and backhand strokes; 2) considering height, BW, wingspan, age, and SL together as independent variables in the application of multiple linear regressions, the height variable does not appear as a predictor of ball speed; 3) the SL is the variable with the greatest independent relationship to ball speed; and 4) the ball speed showed a high absolute and relative reliability.

\section{Acknowledgements}

The authors have no conflicts of interest to declare

\section{Conflict of Interest}

The authors declare that they have no conflict of interest.

\section{References}

[1] Akutagawa S, Kojima T. Trunk rotation torques through the hip joints during the one- and two-handed backhand tennis strokes. J Sports Sci 2005; 23: 781-793

[2] Atkinson G, Nevill AM. Statistical methods for assessing measurement error (reliability) in variables relevant to sports medicine. Sports Med 1998; 26: 217-238

[3] Bonato M, Maggioni MA, Rossi C, Rampichini S, La Torre A, Merati G. Relationship between anthropometric or functional characteristics and maximal serve velocity in professional tennis players. J Sports Med Phys Fitness 2015; 55: 1157-1165

[4] Bower R, Cross R. Elite tennis player sensitivity to changes in string tension and the effect on resulting ball dynamics. Sports Engineering 2008; $11: 31-36$

[5] Brody $\mathrm{H}$. Unforced errors and error reduction in tennis. $\mathrm{Br}$ J Sports Med 2006; 40: 397-400

[6] Caraugh JH, Gabet T, White J. Tennis serving velocity and accuracy. Percept Mot Skills 1990; 70: 719-722

[7] Carlton LG, Chow JW, Shim J. Variability in motor output and Olympic performers. In Davids K, Bennett S, Newell K. (eds.) Movement System Variability. Champaign, IL: Human Kinetics; 2006: 85-108

[8] Cross R, Pollard G. Grand Slam men's singles tennis 1991-2009. Serve speeds and other related data. ITF Coach Sport Sci Rev 2009; 16: 8-10

[9] Chelly SM, Denis C. Leg power and hopping stiffness: Relationship with sprint running performance. Med Sci Sports Exerc 2001; 33: 326-333

[10] di Prampero PE, Fusi S, Sepulcri L, Morin JB, Belli A, Antonutto G. Sprint running: A new energetic approach. J Exp Biol 2005; 208: 2809-2816

[11] Fernández-Fernández J, Sanz-Rivas D, Méndez-Villaneuva A. A review of the activity profile and physiological demands of tennis match play. Strength Cond J 2009; 31: 15-26

[12] Harriss DJ, Macsween A, Atkinson G. Standards for ethics in sport and exercise science research: 2018 update. Int J Sports Med 2017; 38: $1126-1131$

[13] Johnson J. Tennis serve of advanced women players. Res Q Exerc Sport 1957; 28: 123-131

[14] Knudson D, Bahamonde R. Trunk and racket kinematics at impact in the open and square stance tennis forehand. Biol Sport 1999; 16: 3-10
[15] Kolman N, Huijgen B, Kramer T, Elferink-Gemser M, Visscher C. The Dutch technical-tactical tennis test (D4T) for talent identification and development: Psychometric characteristics. J Hum Kinet 2017; 55: 127-138

[16] Kramer T, Huijgen BC, Elferink-Gemser MT, Visscher C. A longitudinal study of physical fitness in elite junior tennis players. Pediatr Exerc Sci 2016; 28: 553-564

[17] Kramer T, Huijgen BC, Elferink-Gemser MT, Visscher C. Prediction of tennis performance in junior elite tennis players. J Sports Sci Med 2017; 16: 14-21

[18] Kramer T, Valente-Dos-Santos ], Coelho ESM], Malina RM, Huijgen BC, Smith J, Elferink-Gemser MT, Visscher C. Modeling longitudinal changes in $5 \mathrm{~m}$ sprinting performance among young male tennis players. Percept Mot Skills 2016; 122: 299-318

[19] Landlinger ], Stöggl T, Lindinger S, Wagner H, Müller E. Differences in ball speed and accuracy of tennis groundstrokes between elite and high-performance players. Eur J Sport Sci 2012; 12: 301-308

[20] Lyons M, Al-Nakeeb Y, Hankey ], Nevill A. The effect of moderate and high-intensity fatigue on groundstroke accuracy in expert and non-expert tennis players. J Sports Sci Med 2013; 12: 298-308

[21] Moore SA, McKay HA, Macdonald H, Nettlefold L, Baxter-Jones AD, Cameron N, Brasher PM. Enhancing a somatic maturity prediction model. Med Sci Sports Exerc 2014

[22] Myburgh GK, Cumming SP, Coelho ESM, Cooke K, Malina RM. Growth and maturity status of elite British junior tennis players. J Sports Sci 2016; 34: 1957-1964

[23] Myburgh GK, Cumming SP, Silva MC, Cooke K, Malina RM. Maturityassociated variation in functional characteristics of elite youth tennis players. Pediatr Exerc Sci 2016; 28: 542-552

[24] Perry AC, Wang X, Feldman BB, Ruth T, Signorile J. Can laboratorybased tennis profiles predict field tests of tennis performance? J Strength Cond Res 2004; 18: 136-143

[25] Reid M, Elliott B, Alderson J. Shoulder joint loading in the high performance flat and kick tennis serves. $\mathrm{Br}$ J Sports Med 2007; 41 : 884-889 discussion 889

[26] Reid MM, Campbell AC, Elliott BC. Comparison of endpoint data treatment methods for estimation of kinematics and kinetics near impact during the tennis serve. J Appl Biomech 2012; 28: 93-98

[27] Sherar LB, Mirwald RL, Baxter-Jones AD, Thomis M. Prediction of adult height using maturity-based cumulative height velocity curves. J Pediatr 2005; 147: 508-514

[28] Stirn I, Carruthers ], Sibila M, Pori P. Frequent immediate knowledge of results enhances the increase of throwing velocity in overarm handball performance. J Hum Kinet 2017; 56: 197-205

[29] Torres-Torrelo J, Rodríguez-Rosell D, González-Badillo JJ. Light-load maximal lifting velocity full squat training program improves important physical and skill characteristics in futsal players. J Sports Sci 2017; 35: 967-975

[30] Ulbricht A, Fernandez-Fernandez ], Mendez-Villanueva A, Ferrauti A. Impact of fitness characteristics on tennis performance in elite junior tennis players. J Strength Cond Res 2016; 30: 989-998

[31] Vaverka F, Cernosek M. Association between body height and serve speed in elite tennis players. Sports Biomech 2013; 12: 30-37

[32] Vergauwen L, Madou B, Behets D. Authentic evaluation of forehand groundstrokes in young low- to intermediate-level tennis players. Med Sci Sports Exerc 2004; 36: 2099-2106

[33] Vincent JW, Weir JP. Statistics in Kinesiology. 4th ed. Champaign, IL: Human Kinetics; 2012

[34] Weir JP. Quantifying test-retest reliability using the intraclass correlation coefficient and the SEM. J Strength Cond Res 2005; 19 : $231-240$ 